Check for
updates

updates

Cite as

Nano-Micro Lett.

(2022) $14: 59$

Received: 24 November 2021

Accepted: 6 January 2022

Published online: 9 February 2022

(C) The Author(s) 2022

\section{Porous and Ultra-Flexible Crosslinked MXene/ Polyimide Composites for Multifunctional Electromagnetic Interference Shielding}

\author{
Zhi-Hui Zeng ${ }^{1}$, Na Wu ${ }^{2}$, Jing-Jiang Wei ${ }^{3}$, Yun-Fei Yang ${ }^{1}$, Ting-Ting $\mathrm{Wu}^{4}, \mathrm{Bin}^{\mathrm{L}}{ }^{1}$, \\ Stefanie Beatrice Hauser ${ }^{4}$, Wei-Dong Yang ${ }^{5}$, Jiu-Rong Liu ${ }^{1}{ }^{\square}$, Shan-Yu Zhao ${ }^{4}$
}

\title{
HIGHLIGHTS
}

- Large-area, lightweight, ultra-flexible, and robust crosslinked MXene-coated PI porous composites were manufactured via a scalable and facile approach.

- In addition to the hydrophobicity, anti-oxidation and extreme-temperature stability, excellent electromagnetic interference shielding performance was achieved because of the high-efficiency utilization of the building units and microstructure.

- Moreover, the highly flexible composite foams exhibited excellent electrothermal and electromechanical sensing performance, demonstrating promising perspectives in next-generation flexible electronics, aerospace, and smart devices.

ABSTRACT Lightweight, ultra-flexible, and robust crosslinked transition metal carbide $\left(\mathrm{Ti}_{3} \mathrm{C}_{2}\right.$ MXene) coated polyimide (PI) (C-MXene@PI) porous composites are manufactured via a scalable dip-coating followed by chemical crosslinking approach. In addition to the hydrophobicity, anti-oxidation and extreme-temperature stability, efficient utilization of the intrinsic conductivity of MXene, the interfacial polarization between MXene and PI, and the micrometer-sized pores of the composite foams are achieved. Consequently, the composites show a satisfactory X-band electromagnetic interference (EMI) shielding effectiveness of 22.5 to $62.5 \mathrm{~dB}$ at a density of 28.7 to $48.7 \mathrm{mg} \mathrm{cm}^{-3}$, leading to an excellent surface-specific SE of $21,317 \mathrm{~dB}$ $\mathrm{cm}^{2} \mathrm{~g}^{-1}$. Moreover, the composite foams exhibit excellent
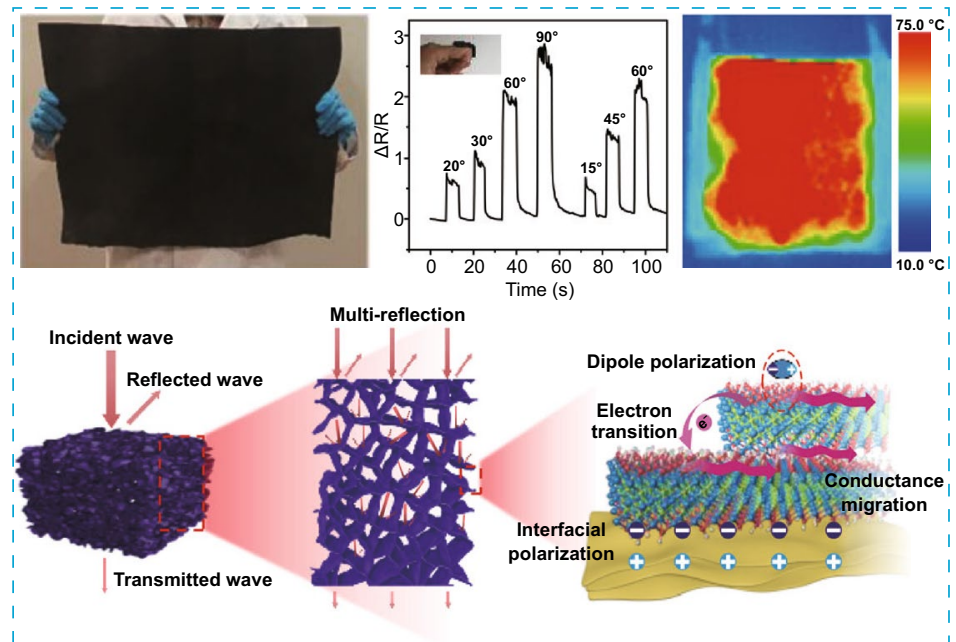

Zhi-Hui Zeng and $\mathrm{Na} \mathrm{Wu}$ have contributed equally to this work

$\triangle$ Zhi-Hui Zeng, zhihui.zeng@sdu.edu.cn; Jiu-Rong Liu, jrliu@sdu.edu.cn; Shan-Yu Zhao, shanyu.zhao@empa.ch

${ }^{1}$ Key Laboratory for Liquid-Solid Structural Evolution and Processing of Materials, Ministry of Education and School of Materials Science and Engineering, Shandong University, Jinan 250061, People's Republic of China

2 Department of Chemistry and Applied Biosciences, ETH Zurich, CH-8093 Zurich, Switzerland

3 Laboratory for Cellulose and Wood Materials, Swiss Federal Laboratories for Materials Science and Technology (Empa), 8600 Dübendorf, Switzerland

${ }^{4}$ Laboratory for Building Energy Materials and Components, Swiss Federal Laboratories for Materials Science and Technology (Empa), 8600 Dübendorf, Switzerland

5 School of Aerospace Engineering and Applied Mechanics, Tongji University, Shanghai 200092, People's Republic of China 
electrothermal performance as flexible heaters in terms of a prominent, rapid reproducible, and stable electrothermal effect at low voltages and superior heat performance and more uniform heat distribution compared with the commercial heaters composed of alloy plates. Furthermore, the composite foams are well attached on a human body to check their electromechanical sensing performance, demonstrating the sensitive and reliable detection of human motions as wearable sensors. The excellent EMI shielding performance and multifunctionalities, along with the facile and easy-to-scalable manufacturing techniques, imply promising perspectives of the porous C-MXene@PI composites in next-generation flexible electronics, aerospace, and smart devices.

KEYWORDS MXene; Polyimide; Electromagnetic interference shielding; Heater; Sensor

\section{Introduction}

Advances in electromagnetic interference (EMI) shielding materials have sparked considerable attention in almost every electronics industry for attenuating electromagnetic radiation of complex electronic systems [1, 2]. High-performance EMI shields with lightweight, superior mechanical flexibility, and improved EMI shielding effectiveness (SE) are urgently required [3]. This promotes the development of numerous EMI shielding composites, involving porous architectures [4-7] or bulk shields [8-10], composed of lightweight, flexible polymers and highly conductive nanomaterials, such as carbon nanotubes (CNTs) [9, 11, 12], metal nanofibers [6, 13], and/or graphene layers [10, 14, 15]. Polymer matrices embedded with varieties of conductive nanofillers give rise to efficient conductive networks and abundant interfaces, which are beneficial for achieving high EMI shielding performance. Particularly, a porous architecture is remarkable in reducing the weight of the shields, and promoting multiple reflections (multi-reflections) of incident EM waves which consequently induces an increased EMI SE [4, 5, 11, 16-18]. For instance, we have reported a type of flexible CNT embedded polyurethane (PU) composite foams with EMI SE of 20-50 dB at density of merely $20-126 \mathrm{mg} \mathrm{cm}^{-3}$ [16]. After compressing the $\mathrm{CNT} / \mathrm{PU}$ foams to exclude the micrometer-sized pores, the corresponding composites show significantly reduced EMI SE [18], confirming the significance of the porous structure in EMI shielding materials. Nevertheless, an efficient dispersion of the inherently inert conductive nanofillers in the polymer matrices accompanied by achieving effective porous structure to obtain high-performance EMI shielding composites in a facile, scalable preparation approach remains a great challenge $[15,19]$.

Transition metal carbides and/or nitrides (MXenes), a novel kind of two-dimensional (2D) nanomaterials, are famous for the "metal-like" conductivity, large specific surface, excellent mechanical properties, and easy processability in aqueous dispersion derived from the hydrophilic functional groups $(-\mathrm{O},-\mathrm{OH},-\mathrm{F})[20,21]$. These provide huge potentials of MXene flakes for bottom-up construction of MXene-based EMI shielding macrostructures [22-24]. However, the interactions formed among MXene flakes are generally very weak, which is against the formation of robust pure MXene porous structures [20, 22, 25], and consequently hinder its practical applications. Polymers acting as efficient 'binder phase' are most widely employed to improve the mechanical strength and flexibility of MXenebased macrostructures [26, 27]. The poly (vinyl alcohol) (PVA) [28], sodium alginate (SA) [22], aramid fiber (ANF) $[29,30]$, and cellulose nanofiber (CNF) [26, 31-34] have been reported to achieve mechanical strong and flexible MXene-based composites. However, the introduced polymers are generally electrical insulator, which inevitably compromise the full utilization of the electrical conductivity and EMI shielding properties of MXenes. Poor temperature tolerance of commonly utilized polymers also restricts the wide range applications of the MXene-based EMI shielding composites [35]. Moreover, the high $\mathrm{H}_{2} \mathrm{O} / \mathrm{O}_{2}$ permeability in polymer-MXene composites is critical for long-term durability regarding the poor oxidation stability of MXene [36-38]. In short, preparing lightweight, flexible, durable MXene-based porous architectures without compromising the excellent electrical conductivity of MXene in a scalable manufacturing approach remains challenging. In addition to EMI shielding performance, integrating multifunctionalities of devices is highly desirable with the rapid development of emerging multifunctional systems with internet of things (IoT) capabilities, such as wearable, flexible electronics including sensors [39-45] and heaters [46-52].

Here, we manufactured the lightweight, flexible, durable, and large-area crosslinked $\mathrm{Ti}_{3} \mathrm{C}_{2}$ MXene-coated polyimide (PI) (C-MXene@PI) composite foams based on a facile and scalable dip-coating followed by chemical crosslinking 
approach. Highly porous yet robust PI scaffolds rendered the composite foams with low density, ultra-flexibility, and extreme-temperatures tolerance. The entire covering of MXene flakes on continuous PI exoskeleton was beneficial for the retainment of high conductivity and EMI shielding performance of pristine MXene. Furthermore, the chemical crosslink of MXene (C-MXene) contributed to the hydrophobicity, waterproof capability, and the oxidation stability of the C-MXene@PI composite foams, promoting their high durability and reliability in practical applications. The 1.5-mm-thick C-MXene@PI composite foams with a density of 28.7 to $48.7 \mathrm{mg} \mathrm{cm}^{-3}$ also exhibited a satisfactory X-band EMI SE of 22.5 to $62.5 \mathrm{~dB}$. Excellent specific SE (SSE, namely SE divided by the density) $[4,9,19]$ and normalized surface-specific SE (SSE/d, namely SE divided by the density and thickness) $[16,22,53]$ values up to 1,971 and $21,317 \mathrm{~dB} \mathrm{~cm}^{2} \mathrm{~g}^{-1}$ were also achieved, respectively, for the C-MXene@PI foams, which are significantly outperforming porous architectures including PI-based composites (Table 1) and other nanofiller embedded polymeric composites (Table S1). Combined with the theoretical calculation, we attributed the excellent EMI shielding performance to the synergistic coactions of micrometer-sized pores, the MXenebased conductive networks, and the interfacial polarization between the MXene and PI. Furthermore, we explored the excellent electrothermal and electromechanical sensing performance of the C-MXene@PI foams, showing the multifunctionalities and the potentials for multifunctional electronic devices. This work thus suggests a convenient, facile approach for large-scale manufacturing high-performance MXene-based porous composites with potential applications in EMI shielding of all kinds of complex electronic systems, and next-generation flexible electronic devices.

\section{Experimental}

\subsection{Materials and Methods}

\subsubsection{Preparation of MXene Aqueous Dispersion}

Aqueous dispersions of MXene were manufactured by etching and mechanical delamination process of the $\mathrm{Ti}_{3} \mathrm{AlC}_{2}$ MAX as shown in our previous work [26]. Briefly, $2.0 \mathrm{~g}$ $\mathrm{Ti}_{3} \mathrm{AlC}_{2}$ MAX (Laizhou Kai Kai Ceramic Materials Co.,
Ltd., China) was added to $40 \mathrm{~mL}$ hydrochloric acid $(\mathrm{HCl}$, $9 \mathrm{M}$, Sigma-Aldrich, the USA) dissolved with $3.2 \mathrm{~g}$ lithium fluoride (LiF, Sigma-Aldrich, the USA). After the reaction at $35{ }^{\circ} \mathrm{C}$ for $24 \mathrm{~h}$, the suspension was centrifuged at $3500 \mathrm{rpm}$ and then redispersed to reach $\mathrm{pH} \approx 6$. Afterward, the suspension was vigorously shaken for $30 \mathrm{~min}$, and then, the supernatant MXene dispersion with a concentration of 0.1 wt $\%$ was obtained.

\subsubsection{Preparation of C-MXene @PI Composite Foams}

PI foams (Solimide ${ }^{\circledR}$ foam, BOYD Corporation $\mathrm{GmbH}$ ) were immersed into the aforementioned MXene dispersion till the MXene dispersion was fully infiltrated into the PI foams and then dried in the $50{ }^{\circ} \mathrm{C}$ oven to get the MXene@ PI composite foams. The dip-coating process was repeated, and the number was recorded. Afterward, the MXene@PI composite foams were chemically crosslinked by PMDI. Here, PMDI was first dissolved in acetonitrile/methyl caproate $(4: 1, \mathrm{v} / \mathrm{v})$ solution at a volume ratio of $1: 9$, and then, the MXene@PI composite foams were immersed in this acetonitrile/methyl caproate $(4: 1, \mathrm{v} / \mathrm{v})$ solution. After a reaction in the oven for $2 \mathrm{~h}$ at $70{ }^{\circ} \mathrm{C}$ followed by a acetone washing treatment, the freestanding C-MXene@PI composite foams were prepared.

\subsection{Characterization}

Scanning electron microscopy (SEM, FEI NanoSEM 230), transmission electron microscopy (TEM, JEOL JEM2200fS) and atomic force microscopy (AFM, Bruker ICON3) were employed to characterize the morphology and microstructure. A drop shape analyzer (DSA 30, Krüss, Germany) was utilized to measure the water contact angles (CA). A FTIR spectrometer (PerkinElmer Spectrum Two) with an attenuated total reflection accessory was used to perform the FTIR measurements. The resistances $(R)$ were measured in a four-probe method by a Keithley 4200 electrometer so as to calculate the electrical conductivity $(\delta)$. EMI SE in the frequency range of 8.2-12.4 GHz (X-band) was measured by a vector network analyzer (Agilent 8517A) in the waveguide method. More than three specimens were tested for each component. The S-parameters were recorded and used to calculate the 
Table 1 EMI shielding performance of PI-based macrostructures and some typical MXene composites

\begin{tabular}{|c|c|c|c|c|c|c|}
\hline Materials & EMI SE (dB) & Density $\left(\mathrm{mg} \mathrm{cm}^{-3}\right)$ & Thickness (mm) & $\operatorname{SSE}\left(\mathrm{dB} \mathrm{cm} \mathrm{g}^{3} \mathrm{~g}^{-1}\right)$ & $\mathrm{SSE} / d\left(\mathrm{~dB} \mathrm{~cm} \mathrm{~g}^{2}\right)$ & Refs \\
\hline \multirow[t]{7}{*}{ C-MXene@PI foam } & 43.7 & 41.0 & 0.5 & 1066 & 21,317 & \multirow[t]{7}{*}{ This work } \\
\hline & 80.8 & 41.0 & 3 & 1971 & 6569 & \\
\hline & 62.52 & 48.7 & 1.5 & 1285 & 8567 & \\
\hline & 60.04 & 43.0 & & 1397 & 9315 & \\
\hline & 59.17 & 41.0 & & 1442 & 9612 & \\
\hline & 52.51 & 38.0 & & 1383 & 9217 & \\
\hline & 45.54 & 35.6 & & 1278 & 8519 & \\
\hline Ag NWs/PI foam & $17-23.5$ & 22 & 5 & $1068-772$ & $2136-1544$ & [59] \\
\hline CNT/PI foam & 41.1 & 32.1 & 2 & 1280 & 6402 & {$[60]$} \\
\hline MWCNT/PI & $13.0-14.3$ & 470 & 0.5 & $28-30$ & $553-609$ & [69] \\
\hline $\mathrm{rGO} / \mathrm{PI}$ foam & $13.7-15.1$ & 460 & 0.5 & $30-33$ & $596-657$ & [69] \\
\hline MWCNT-CNT/rGO/PI foam & $16.6-18.2$ & 440 & 0.5 & $38-41$ & $755-823$ & [69] \\
\hline CNT/graphene/PI foam & 28.2 & 20 & 2 & 1410 & 7050 & {$[70]$} \\
\hline Graphene/PI foam & 22 & 280 & 0.8 & 78.6 & 982 & {$[63]$} \\
\hline Anisotropic graphene/PI foam & $26.1-28.8$ & 76 & 2.5 & $343-379$ & $1373-1518$ & [71] \\
\hline Graphene/PI foam & $13.7-14.9$ & 430 & 0.5 & $32-35$ & $637-693$ & [72] \\
\hline Graphene/PI film & 31.3 & $\sim 1200$ & 0.151 & 26 & 1727 & [73] \\
\hline Carbon nanofiber/PI film & 12 & $\sim 1200$ & 0.07 & 10 & 1429 & [74] \\
\hline $\begin{array}{l}\text { Carbon nanofiber/carbon black/ } \\
\text { PI film }\end{array}$ & 23.9 & $\sim 1200$ & 0.35 & 20 & 571 & {$[75]$} \\
\hline PI derived carbon foam & 54 & 91 & 2 & 593 & 2965 & [76] \\
\hline Graphene/PI-derived carbon foam & 24 & 720 & 0.024 & 33 & 13,888 & [77] \\
\hline MXene/PI porous film & 54.5 & 390 & 0.09 & 140 & 15,527 & [78] \\
\hline MXene/nanocellulose film & 24 & 2000 & 0.047 & 12 & 2647 & {$[31]$} \\
\hline MXene/CNF film & 33 & 2477 & 0.0009 & 37 & 148,000 & {$[57]$} \\
\hline MXene/CNF foam & 75 & 0.008 & 2 & 9320 & 46,600 & {$[26]$} \\
\hline MXene/PVA porous film & 26 & $\sim 545$ & 0.1 & 48 & 4770 & {$[28]$} \\
\hline MXene/PVA foam & 28 & 0.0108 & 5 & 2586 & 5136 & {$[28]$} \\
\hline MXene/ANF & 28 & 1250 & 0.02 & 22 & 11,200 & [29] \\
\hline MXene/SA film & 57 & $\sim 2317$ & 0.008 & 25 & 30,830 & [22] \\
\hline
\end{tabular}

$\mathrm{SE}_{\mathrm{T}}, \mathrm{SE}_{\mathrm{R}}$, and $\mathrm{SE}_{\mathrm{A}}$. To evaluate the electrothermal performance, various DC voltages were applied to the $10 \mathrm{~L}$ MXene@PI composite foams using a DC-regulated power supply. The temperature of the sample was measured by a digital thermometer (UT325) with its T-type thermocouple contacting the surface of the sample. The electromechanical response of the composite foams was obtained by measuring the resistance change using the Keithley 4200SCS electrometer in a two-probe method.

\section{Results and Discussion}

\subsection{Preparation and Structure of the Composite Foam}

The manufacturing process of C-MXene@PI composite foams is schematically displayed (Fig. 1a, b). First, a stable $\mathrm{Ti}_{3} \mathrm{C}_{2} \mathrm{~T}_{\mathrm{x}}$ MXene (T represents the surface hydrophilic functional groups (-OH, $-\mathrm{O}$, and $-\mathrm{F})$ ) in an aqueous dispersion was prepared by etching and delamination of the precursor 
$\mathrm{Ti}_{3} \mathrm{AlC}_{2} \mathrm{MAX}$ with a compact rocklike microstructure (Figs. 1a and S1a) [26, 27]. After etching of Al layers of MAX precursor [26, 54], multilayer $\mathrm{Ti}_{3} \mathrm{C}_{2} \mathrm{~T}_{\mathrm{x}}\left(\mathrm{m}-\mathrm{Ti}_{3} \mathrm{C}_{2} \mathrm{~T}_{\mathrm{x}}\right)$ was obtained (Fig. S1b). Subsequent repeated washing with deionized water and vigorous shaking to swell the $\mathrm{m}-\mathrm{Ti}_{3} \mathrm{C}_{2} \mathrm{~T}_{\mathrm{x}}$ were carried out, leading to the preparation of aqueous dispersion composed of delaminated MXene nanoflakes. A high Zeta potential of around $-40 \mathrm{mV}$ showed stability of the MXene in aqueous dispersion (Fig. S1c). Dominant single-layer MXene flakes with an average lateral size of around $2 \mu \mathrm{m}$ and a hexagonal atomic structure were observed in the TEM and electron diffraction images, respectively (Fig. 1c). AFM image is further provided, and the thickness of $\sim 1.7 \mathrm{~nm}$ can be identified for a single MXene flake (Fig. 1d); this is consistent with the previous reports [26, 27, 55]. The pure PI foams showed micrometersized pores and smooth pore cell surfaces (Fig. S2a), which interconnected and sustained the low-density $\left(\sim 25 \mathrm{mg} \mathrm{cm}^{-3}\right)$ porous scaffolds. The strong hydrogen bonding interactions formed between the imide rings of PI and MXene nanoflakes allowed for MXene adhered well to PI skeletons, leading to a successful preparation of MXene-coated PI (MXene@PI) composite foams. The coated MXene flakes interconnected to form substantial conductive networks, which were instrumental in significantly improving the electrical conductivity of the PI scaffolds [56]. The MXene nanoflakes were

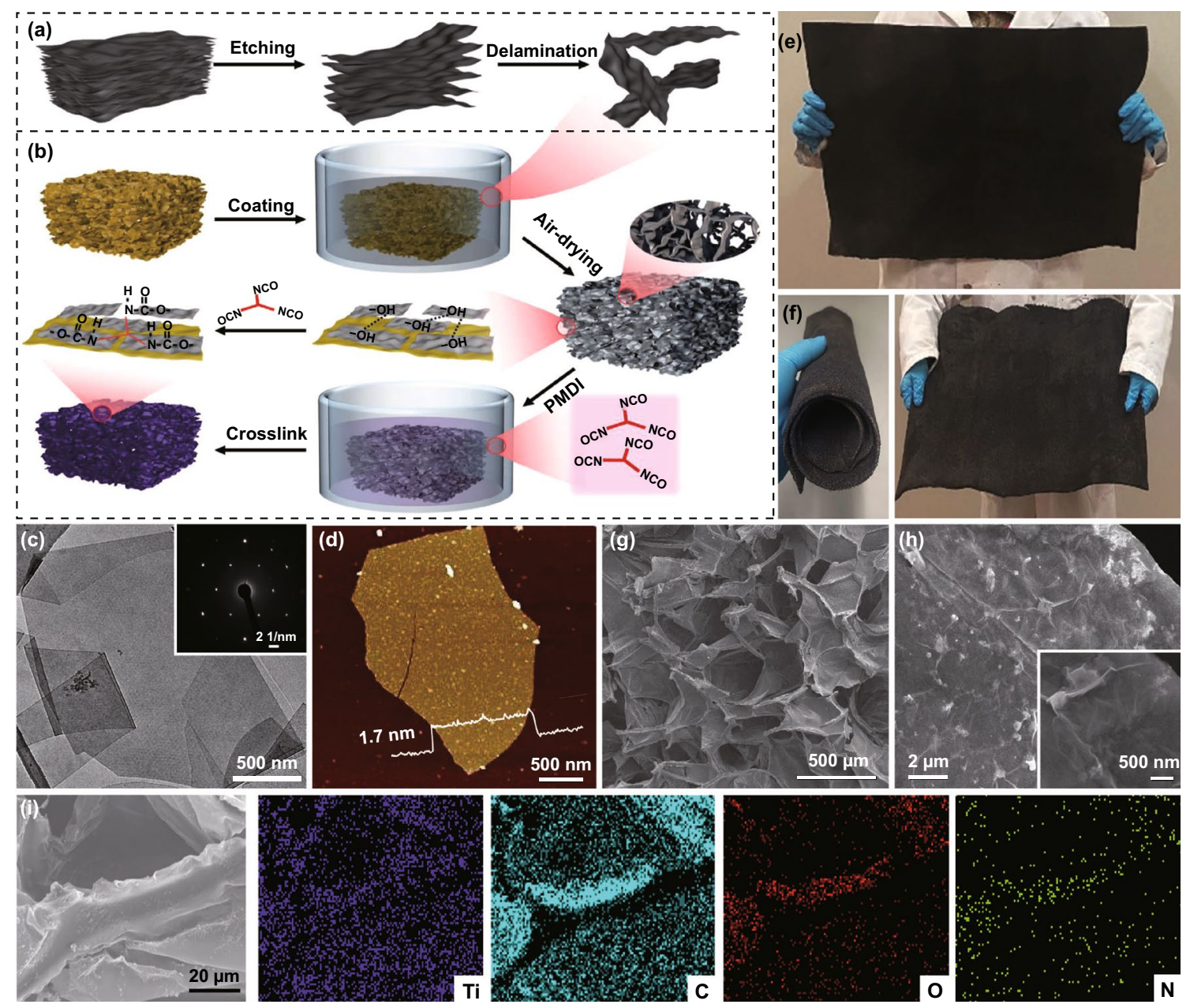

Fig. 1 Scalable manufacturing of C-MXene@PI composite foams: schematic of preparation process of a MXene flakes and b C-MXene@PI composite foams, c TEM (inset shows electron diffraction image) and d AFM (inset shows the height profile of a monolayer) images of the MXene flakes. e, f Photographs of freestanding and large-area $\left(\sim 60 \times 60 \mathrm{~cm}^{2}\right)$ C-MXene@PI composite foams before and after rolling, showing the mechanical robustness and flexibility. SEM images of the $\mathbf{g}$ micrometer-sized pores and $\mathbf{h}$ cell walls, and $\mathbf{i}$ Ti-, C-, O-, and N- element mappings of the C-MXene@PI composite foams 
further crosslinked by the chemical crosslinking agent poly ((phenyl isocyanate)-co-formaldehyde) (PMDI). The isocyanate group of PMDI efficiently reacts with the hydroxyl groups on MXene, leading to the formation of strong peptide bonds. A typical, large-area $\left(60 \times 60 \mathrm{~cm}^{2}\right)$ crosslinked MXene-coated PI (C-MXene@PI) composite foams exhibiting excellent mechanical robustness and flexibility were manufactured (Fig. 1e, f). Compared with the pure PI foams, the C-MXene@PI foams showed the same micrometer-sized pores yet rougher cell walls due to the crosslinked MXene layers on the PI cell walls (Figs. 1g, h and S2b). Element mappings of the C-MXene@PI composite foams further demonstrated the successful composite and that the pore walls were coated with numerous MXene flakes (Fig. 1i).

The interfacial interactions between MXene and PI as well as the flexible and robust PI scaffolds endowed the C-MXene@PI composite foams with excellent mechanical flexibility including bendability, rollability, twistability, and even foldability (Fig. 2a). Even though soaked in the liquid nitrogen at an extreme temperature of $-196{ }^{\circ} \mathrm{C}$, the ultra-flexibility of the C-MXene@PI composite foam was still maintained (Fig. 2b, Video S1). In contrast, the commercial PU foams upon bending broke easily in this low-temperature condition (Fig. S3, Video S2), proving the significance of PI scaffolds on our flexible and reliable composite foams especially for extreme conditions. Furthermore, the chemical crosslinking of MXene flakes increased the water contact angle from $0^{\circ}$ to $118^{\circ}$ (Figs. $2 \mathrm{c}$ and S4a, Video S3), which was mainly ascribed to the introduction of the hydrophobic backbone in the PMDI [57]. As displayed in FTIR curves (Figs. 2d and S5), the characteristic peaks of polyimide $\left(1720,1780 \mathrm{~cm}^{-1}\right.$ for $\mathrm{C}=\mathrm{O}$ and $1380 \mathrm{~cm}^{-1}$ for $\mathrm{C}-\mathrm{N})$ presented in both pure polyimide and MXene-coated polyimide foams (Fig. 2d). After incubated with chemical crosslinker PMDI, the emblematic bands (around 1410 and $1504 \mathrm{~cm}^{-1}$ ) of benzene ring in PMDI can be observed on the spectra of C-MXene@PI (Fig. S5a). The new appearance of characteristic benzene ring vibrations and a $\mathrm{CO}-\mathrm{NH}$ mode (1700 $\mathrm{cm}^{-1}$ for $\mathrm{C}=\mathrm{O}$ in the urethane bonding, Fig. S5b) in C-MXene@PI showed the successful chemical coating of PMDI on the MXene. Consequently, such hydrophobic coating led to excellent stability and water resistance of the MXenes composites. After ultrasonic treatment for $20 \mathrm{~min}$ of the MXene@PI and C-MXene@PI composite foams were immersed in water, the former deteriorated completely, while the latter stayed as one piece, and there was no MXene detachment (Figs. 2e and S4b).

\subsection{EMI Shielding Performance of the Composite Foam}

In addition to the significant color change of the PI scaffolds upon the coating of MXene nanoflakes (Fig. S6), the 2-theta angle of $\sim 7.22^{\circ}$ in XRD pattern corresponding to the interlayer gaps of $1.2 \mathrm{~nm}$ between the MXene nanoflakes further shows a well-preserved structure of MXene flakes (Fig. 2f), which indicates a high utilization efficiency of MXenes' conductivity and EMI shielding properties [22]. The coated MXene flakes on PI skeleton led to a remarkable increase in X-band EMI SE from 1.4 to $60 \mathrm{~dB}$ for the MXene@PI composite foams at a thickness of $1.5 \mathrm{~mm}$. It is worth noting that EMI SE describes the attenuation capability of samples to the incident EM waves (Table S2), and an EMI SE value of $20 \mathrm{~dB}$ corresponding to a $99 \%$ attenuation of the waves is generally required for commercial applications [13, 19, 22]. Apart from the satisfactory EMI SE, our composite foams showed larger SE values than other PI-based composites (Table 1) and typical conductive nanomaterial embedded porous composites ever reported at similar thicknesses [58], e.g., 5-mm-thick AgNW/PI [59], 2-mm-thick CNT/PI [60], 2.3-mm-thick CNT/PU [16], 2.3-mm-thick graphene/PEI [61] composite foams reached SE values up to 12.8, 41.1, 50.5 , and $12.8 \mathrm{~dB}$, respectively. Chemical crosslinking of MXene flakes shown ignorable influence on the electrical conductivity and EMI SE properties (Figs. 2g and S7a), demonstrating the potentials of our C-MXene@PI composite foams as high-performance EMI shielding materials. More intriguingly, the chemical crosslink efficiently improved the oxidation stability of the C-MXene@PI composites in $\mathrm{H}_{2} \mathrm{O} / \mathrm{O}_{2}$ environment (Fig. 2h-i), which is crucial for long durability of the composites in practical applications. After being stored in a $95 \% \mathrm{RH}$ environment and a temperature of $60{ }^{\circ} \mathrm{C}$ for 2 days, EMI SE of the MXene@PI composite foams remarkably decreased and the shielding effect almost disappeared after 6 days in the same condition. In contrast, the C-MXene@PI composite foams maintained a high EMI SE of $44.4 \mathrm{~dB}$ after being stored in the same condition for 6 days (Fig. 2j). The behavior for resistance change of the composite foam stored in such condition is consistent with that of EMI SE (Fig. S7b). Therefore, stable, durable 

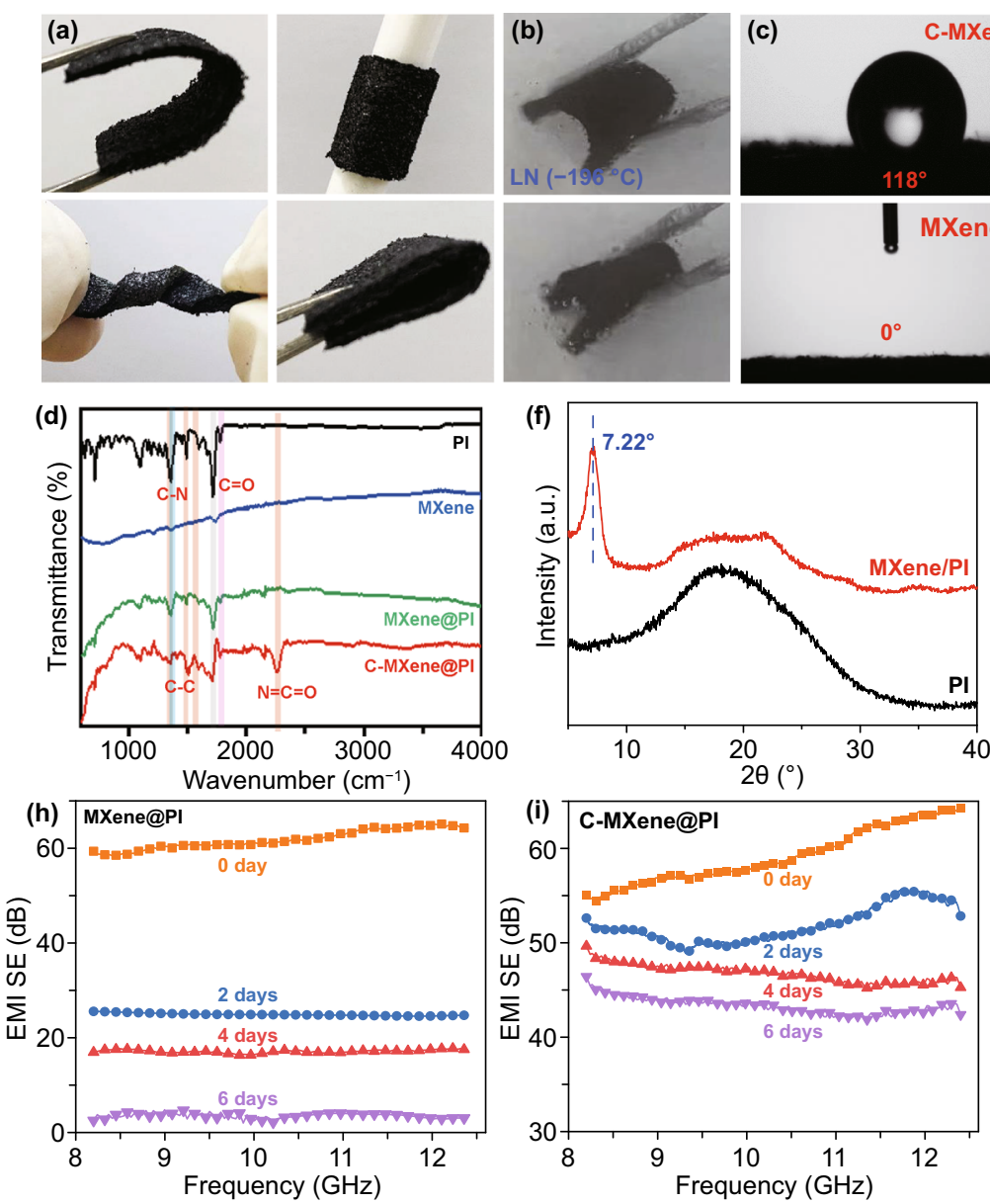
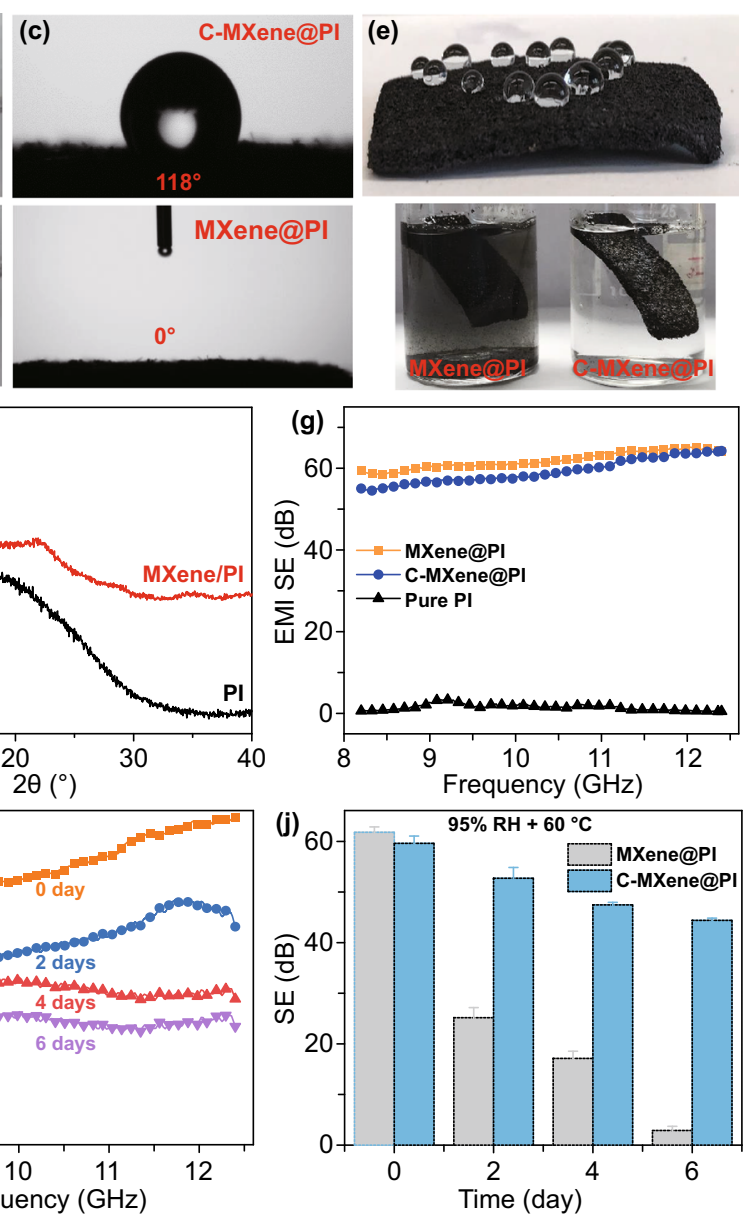

Fig. 2 Photographs of a the C-MXene@PI composite foams showing ultra-flexible performance including bendability, rollability, twistability, foldability at room temperature and $\mathbf{b}$ the composite soaked in liquid nitrogen (LN, $-196{ }^{\circ} \mathrm{C}$ ) with maintained mechanical flexibility. $\mathbf{c}$ Contact angles of the MXene@PI and C-MXene@PI composite foams.d FTIR curves of PI, MXene@PI, and C-MXene@PI foams. e Photograph of C-MXene@PI foams with several drops of water on the surface, as well as the MXene@PI and C-MXene@PI foams after ultrasonic treatment in water for $30 \mathrm{~min}$, showing that the C-MXene@PI foams are waterproof. f XRD patterns of the PI and C-MXene@PI foams. g X-band EMI SE of the PI, MXene@PI, and C-MXene@PI foams. X-band EMI SE of the h MXene@PI and i C-MXene@PI composite foams after stored in a $95 \% \mathrm{RH}$ environment and a temperature of $60{ }^{\circ} \mathrm{C}$ for different days and $\mathbf{j}$ the corresponding change of SE as a function of time. In the first day $(d=0)$, the foams were in a dry state

C-MXene@PI composite foams with a remarkable EMI SE were achieved successfully.

In the facile and scalable "layer-by-layer" dip-coating approach, EMI SE of C-MXene@PI composite foams can be widely controlled by adjusting the coating layers/times with MXene suspensions (Fig. 3a). The EMI SE of the 1.5 -mm-thick porous composites increased with increasing coating layers, e.g., EMI SE reached a commercial SE value after a 4 times coating (namely 4 Layers MXene, $4 \mathrm{~L}$ ), while it increased to 41 and $60 \mathrm{~dB}$ after 10 and 16 times coatings, respectively. Nevertheless, when the composites were coated by $18(18 \mathrm{~L})$ and more times, the increase of EMI SE reached a plateau. To better realize the behavior, we concluded the density and electrical conductivity of the C-MXene@PI composite foams as a function of coating layer (Fig. 3b). The density increased with the increasing of the coating layers, which correlates to the increased MXene loading and related thickness on PI scaffolds. According to the MXene-PDMI layer thickness (around $0.8 \mu \mathrm{m}$ ) identified from the SEM image of a 14L C-MXene/PI composite foam (Fig. S2b), we can easily calculate the MXene-PDMI thickness on the PI skeletons based on the measured density of the composite foams. Furthermore, a clear observation in Fig. 3a, 1L C-MXene@PI composite foams can already form sufficient conductive paths due to the efficient adhesion of MXene on the interconnected PI cell walls. This led to the 

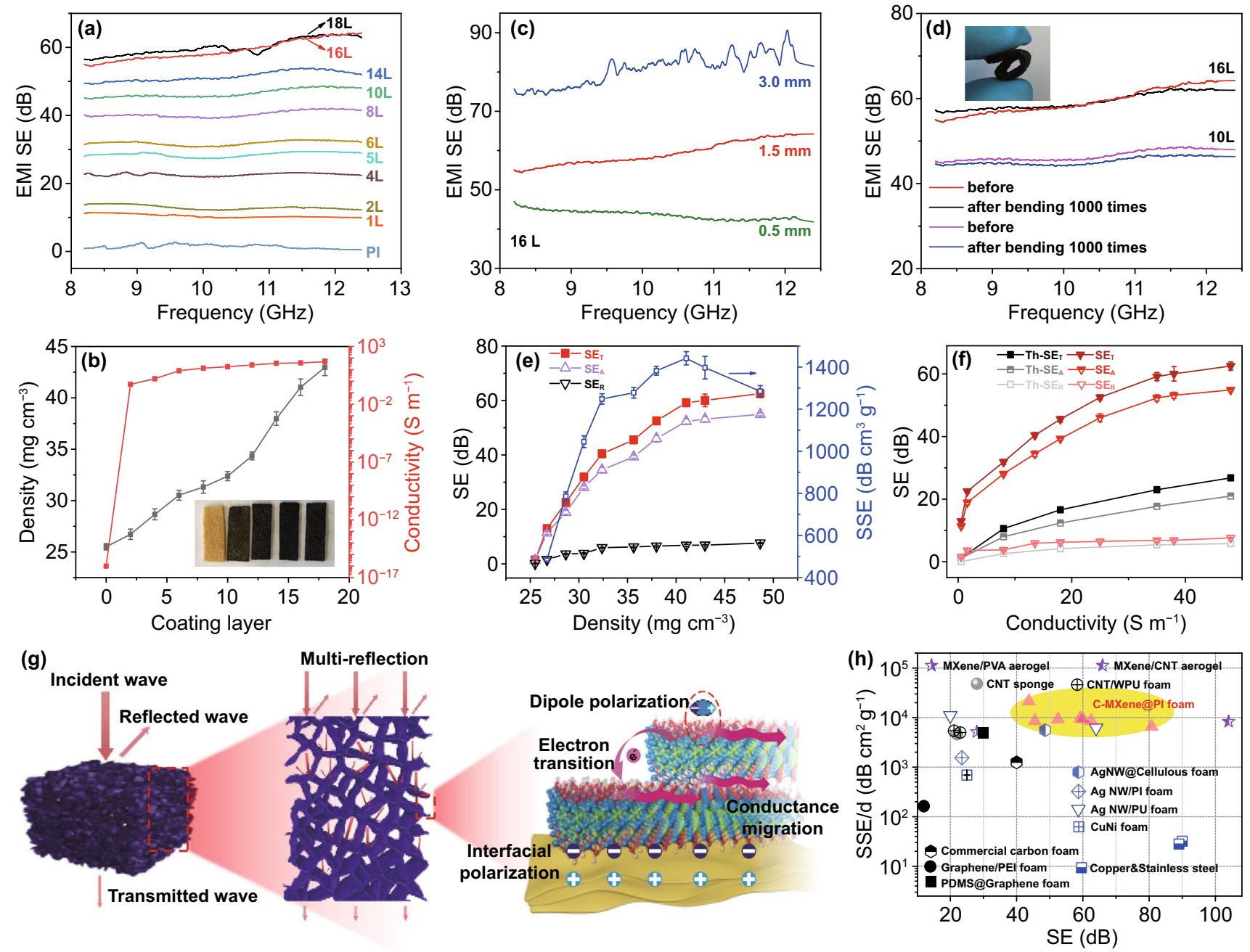

Fig. 3 a X-band EMI SE of C-MXene@PI composite foams with various dip-coating layers. b Density and electrical conductivity of the C-MXene@PI composite foams as a function of the dip-coating layers. c The 16L C-MXene@PI composite foams at various thicknesses, and d the 10L and 16L C-MXene@PI composite foams before and after 1000 times bending treatment, showing the good shielding stability against mechanical deformations. e EMI shielding performance $\left(\mathrm{SE}_{\mathrm{T}}, \mathrm{SE}_{\mathrm{A}}\right.$, and $\left.\mathrm{SE}_{\mathrm{R}}\right)$ and SSE of C-MXene@PI composite foams as function of sample density. $\mathbf{f}$ Theoretically calculated EMI shielding performance (Th-SE $\mathrm{T}, \mathrm{Th}-\mathrm{SE}_{\mathrm{A}}$, and Th-SE $\mathrm{R}_{\mathrm{R}}$ ) of a bulk based on the electrical conductivity of corresponding C-MXene@PI foams and the comparison to the experimentally tested EMI shielding performance $\left(\mathrm{SE}_{\mathrm{T}}, \mathrm{SE}_{\mathrm{A}}\right.$, and $\left.\mathrm{SE}_{\mathrm{R}}\right)$ of the C-MXene@PI composite foams. g Schematic showing the proposed EMI shielding mechanism of the C-MXene@PI composite foams for ultrahigh EMI shielding performance. h EMI shielding performance comparison of the C-MXene@PI composite foams with other typical porous architectures: SSE/d values of typical EMI shielding macrostructures with corresponding SE values (the experimental data and source reference used in this figure are summarized in Table S1)

rapid transformation from insulation to conduction of the porous scaffolds. With increasing of coating layers, more MXene nanoflakes were stacked on the cell walls, forming improved conductive networks, which eventually affected the electrical conductivity and EMI SE of the composites. However, the changes of electrical conductivity, as well as EMI SE, were not remarkably, especially above $10 \mathrm{~L}$ coatings, which indicates good compatibility between MXene and PI fibers, and abundant intact conductive paths have been well established during the initial several coating rounds. Additionally, the EMI SE was controlled by adjusting the thickness of the C-MXene@PI composite foams, e.g., 16L composites reached an EMI SE value of 43.7 to $80.8 \mathrm{~dB}$ at a thickness of 0.5 to $3.0 \mathrm{~mm}$ (Fig. 3c). Moreover, the porous composites showed excellent resistance and EMI SE stability upon mechanical deformation, the resistance 
and EMI SE remained almost constant after the sample was bent for 1000 cycles (Figs. 3d and S7c). Briefly, the controllable and stable EMI shielding performance further demonstrates the great promises of our C-MXene@PI foams for practical applications.

In order to realize the EMI shielding mechanism of the C-MXene@PI composite foams, we measured the shielding by absorption $\left(\mathrm{SE}_{\mathrm{A}}\right)$ and reflection $\left(\mathrm{SE}_{\mathrm{R}}\right)$ (Fig. 3e). Generally, EMI shielding performance of conductive porous composites is influenced by the reflection, absorption, and multi-reflections, corresponding to the mobile charge carriers, electric dipoles, and interior interfaces/surfaces, respectively $[19,56,62]$. The micrometer-sized pores induced more reflections or scatterings of incident EM waves, which had more interactions with the pore walls in the C-MXene@ PI composite foams, efficiently increasing $\mathrm{SE}_{\mathrm{A}}$ [16-18, 63]. The large mismatch of conductivity in the interfaces between the MXene and PI also led to high interfacial polarization [8], which combined with the abundant charge carriers from MXenes [23, 63, 64], resulting in increased $\mathrm{SE}_{\mathrm{A}}$ of the pore walls. In addition, the MXene terminal functional groups were considered to give rise to electric dipoles under the electric field of the EM wave $[17,22,26]$, improving the $\mathrm{SE}_{\mathrm{A}}$ of the MXene-based composites. Thus, the $\mathrm{SE}_{\mathrm{A}}$ dominated the total $\mathrm{SE}\left(\mathrm{SE}_{\mathrm{T}}\right)$, which sum up both $\mathrm{SE}_{\mathrm{R}}$ and $\mathrm{SE}_{\mathrm{A}}$. With increasing density of the C-MXene@PI composite foams derived from increased $\mathrm{MXene}$ loadings, $\mathrm{SE}_{\mathrm{T}}$ and $\mathrm{SE}_{\mathrm{A}}$ of the composites thus increased significantly and achieved maximum values of 62.5 and $54.9 \mathrm{~dB}$, respectively, at a density of $48.7 \mathrm{mg} \mathrm{cm}^{-3}$. Furthermore, we had theoretically calculated the EMI shielding performance $\left(\mathrm{Th}-\mathrm{SE}_{\mathrm{T}}, \mathrm{Th}-\mathrm{SE}_{\mathrm{A}}\right.$, and Th-SE $E_{R}$ ) (the details of the theoretical calculation method are shown in our previous work $[13,16])$ of a homogenous shield based on the conductivities derived from our C-MXene@PI composite foams (Fig. 3f). Obviously, apart from the similar $\mathrm{SE}_{\mathrm{R}}$ values, $\mathrm{Th}-\mathrm{SE}_{\mathrm{A}}$ was obviously lower than the experimentally tested $\mathrm{SE}_{\mathrm{A}}$. This was attributed to that the introduced multi-reflections caused by the porous structure gave rise to more interactions of the incident waves with the MXene/PI composite cell walls, which effectively absorbed the EM waves derived from the conduction and polarization loss capability. As a consequent, the experimentally tested $\mathrm{SE}_{\mathrm{T}}$ was much higher than the $\mathrm{Th}-\mathrm{SE}_{\mathrm{T}}$. In short, we could efficiently demonstrate that the high EMI shielding performance of our C-MXene@PI composite foams is attributed to the synergistic interactions among MXene, PI skeleton and the porous structure (Fig. 3g).

To better realize the lightweight EMI shielding architectures, we calculated the SSE of the C-MXene@PI composite foams with various densities (Fig. 3e). Interestingly, the SSE initially had a significant increase with increasing density, and it reached the extremum of $1,442 \mathrm{~dB} \mathrm{~cm}^{3} \mathrm{~g}^{-1}$ at a density of $41 \mathrm{mg} \mathrm{cm}^{-3}$, and a further increase in density led to the drop of SSE. This illustrated that suitable coating times of the MXene nanoflakes on the PI skeleton were vital for better utilizing the MXene for the EMI shielding porous architectures. Notably, the EMI SE reached a value of $59.2 \mathrm{~dB}$ at such a large SSE for the C-MXene@PI composite foams, which showed opposite behavior with other composites having a higher SSE value with decreasing density or SE values $[5,16,18]$. Here, the efficient design of our C-MXene@PI composite foams contributed both higher SE and SSE values, which significantly outperformed that of other typical nanofiller embedded polymeric porous composites at similar thickness (Table 1), e.g., EMI SE was around 22, 41, and $23 \mathrm{~dB}$ for the graphene/PI [65], CNT/PI [58], and CNT/ PU [16] porous composites at SSE values of 78.6, 1280, and $1184 \mathrm{~dB} \mathrm{~cm}^{3} \mathrm{~g}^{-1}$, respectively. Certainly, a higher SE associated with a minimum material consumption is crucial for achieving high-performance EMI shielding architectures, and thus, SSE/d was proposed in our previous work [13, $16,53]$ to efficiently evaluate the lightweight EMI shields. Herein, the SSE/ $d$ of C-MXene@PI composite foams could reach 9,612 and $21,317 \mathrm{~dB} \mathrm{~cm}^{2} \mathrm{~g}^{-1}$ at a $\mathrm{SE}$ of 59.2 and $43.7 \mathrm{~dB}$, respectively. This performance was superior to that of other porous EMI materials including commercial carbon foams, carbon nanotube sponges, graphene-based foams, metal-based foams, and other MXene-based porous composites (Fig. 3h, Table S1). Particularly, the EMI shielding performance among the PI-based architectures is compared in Table 1, effectively proving the superiority of EMI performance of C-MXene@PI composite foams. Combined with the service stability, as well as the energy-efficient, facile, and scalable preparation method, the lightweight, ultra-flexible, and robust C-MXene@PI composite foams with outstanding EMI shielding performance show huge potentials in the applications of aerospace, portable electronics and smart wearable devices. 


\subsection{Electrothermal Performance of the Composite Foam}

Besides the EMI application, multifunctionality is always expected for such lightweight conductors. First extension was on electrothermal materials, which convert electric power to thermal energy have attracted more and more attention due to the rapid development of electronic engineering [47, 48, 51, 52]. Traditional electrothermal materials, including $\mathrm{Fe}-\mathrm{Cr}-\mathrm{Al}$ or $\mathrm{Ni}-\mathrm{Cr}$-based alloys and electrothermal ceramics, suffer from complicated manufacturing process, heavy weight, inflexible shape and low heating efficiency. Here, thermogravimetric analysis (TGA) curves of pure PI and C-MXene@PI foams showed the good thermal stability of the PI-based architectures (Fig. 4a), in contrast to most polymers. Combined with the efficient conductive networks, the ultra-flexible C-MXene@PI composite foams showed great potentials for lightweight and stable electrothermal heaters. Therefore, various DC voltages were applied to the C-MXene@PI composite foams $\left(4 \times 4 \times 0.15 \mathrm{~cm}^{3}\right)$, and the currents flowing through the foams were observed. Figure $4 \mathrm{~b}$ showed the effect of these low DC voltages on the temperature of the 10L C-MXene@PI composite foams. The composites had evident, stable, and reversible electrothermal effect at these voltages. For example, the composite foams with a large size of $40 \times 40 \times 1.5 \mathrm{~mm}^{3}$ were able to reach up to $34,53,84$, and $114{ }^{\circ} \mathrm{C}$ in tens of seconds at $4,6,8$, and $10 \mathrm{~V}$, respectively. At the same time, the life-time tests for the composite heaters had been carried out. As shown in Fig. 4c, a DC voltage of $6 \mathrm{~V}$ was applied to the composite
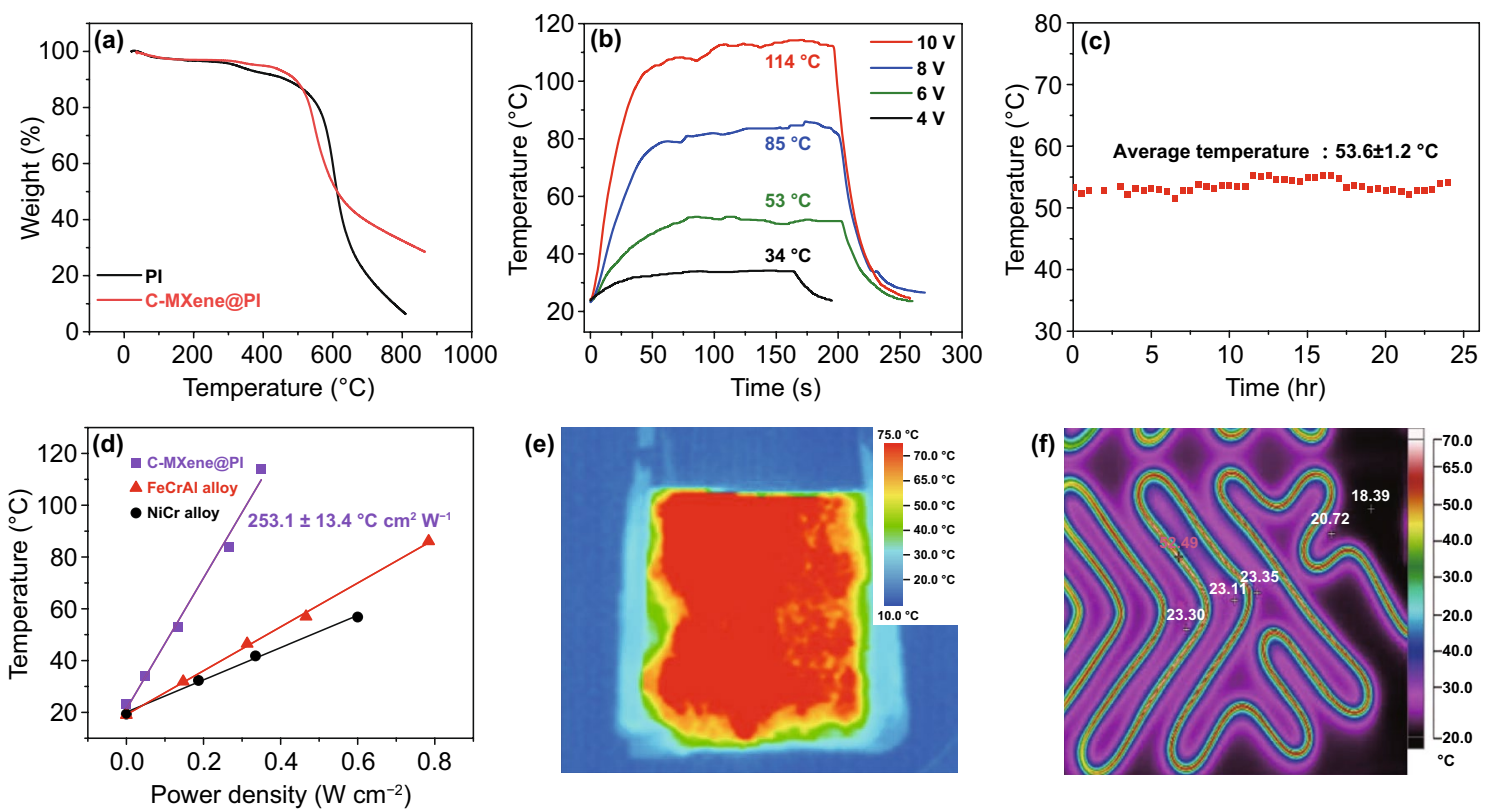

(e)
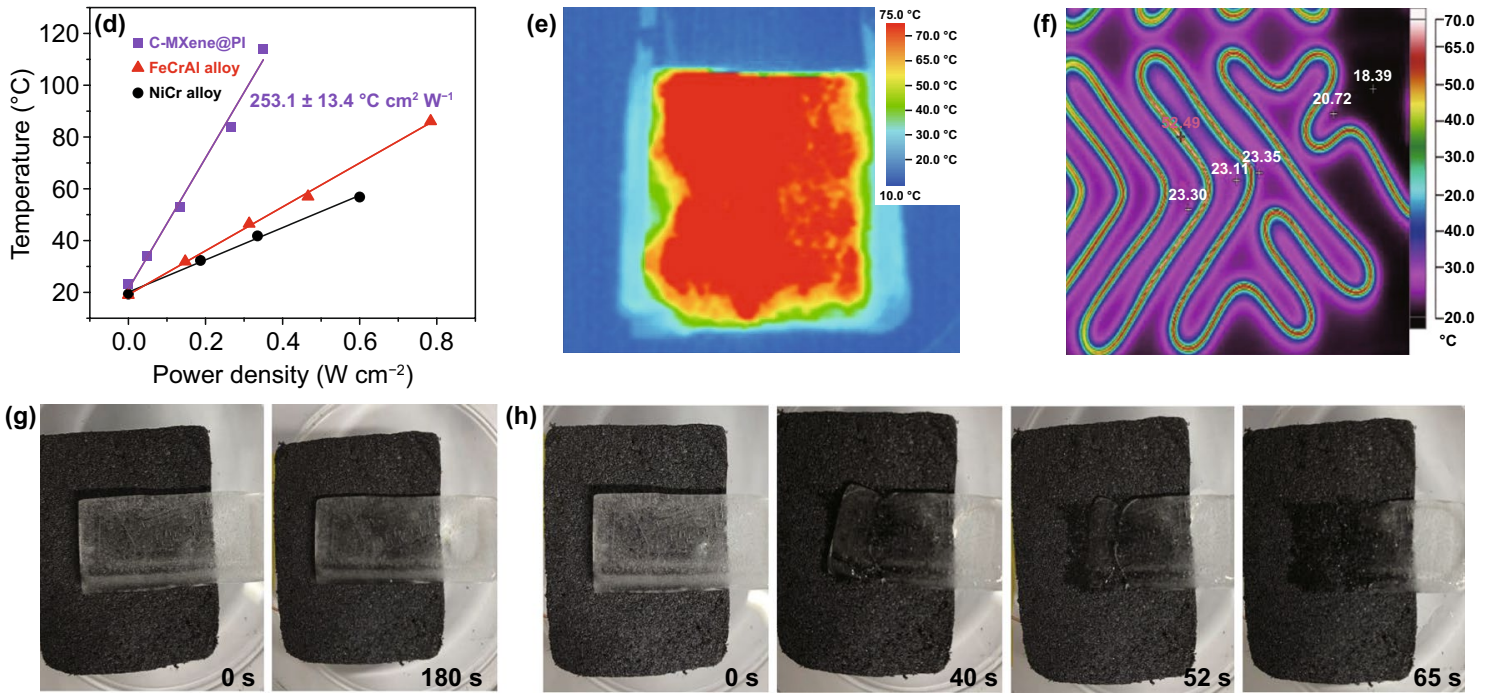

Fig. 4 Electrothermal performance of the C-MXene@PI composite foams. a TGA curves of the C-MXene@PI composite and pure PI foams in air, b the electrothermal curves of the C-MXene@PI composite foams $\left(4 \times 4 \times 0.15 \mathrm{~cm}^{3}\right)$ at various voltages, and $\mathbf{c}$ life-time tests of the C-MXene@PI composite foams for $24 \mathrm{~h}$. d The steady-state temperature versus input power density curves of the C-MXene@PI composite foams and commercial $\mathrm{Fe}-\mathrm{Cr}-\mathrm{Al}$ and $\mathrm{Ni}-\mathrm{Cr}$ alloy plates. Thermal image map of the e C-MXene@PI composite foams and $\mathbf{f}$ commercial metalbased heaters, showing the uniform temperature of the C-MXene@PI composite foams. Electrothermal deicing performance. Demonstration for a deicing application of the C-MXene@PI composites: $\mathbf{g}$ the composite without applied voltage and $\mathbf{h}$ the composite at an applied voltage of $8 \mathrm{~V}$ 
heaters for $24 \mathrm{~h}$, and the temperature was measured every half hour. It was found that the steady-state heating of the samples, which further demonstrated a stable electrothermal effect of the composite. A stable and remarkable electrothermal performance of the C-MXene@PI composite foams was achieved at low DC voltages, demonstrating the possibility and prospects in various application areas.

The equilibrium temperature of the composites showed a linear relation with the input power densities (Fig. 4d). The slope of the steady-state temperature versus input power density revealed the heat performance $\left(H_{\mathrm{p}}=\mathrm{d} T / \mathrm{d} P\right)$ of the composite heater $[53,56]$, as the greater the $H_{\mathrm{p}}$ value, the higher the steady-state temperature of the heater, at certain initial temperature and input electric power. The $H_{\mathrm{p}}$ value of the C-MXene@PI composite samples was $253.1{ }^{\circ} \mathrm{C}$ $\mathrm{cm}^{2} \mathrm{~W}^{-1}$, higher than that of other systems including CNTs, rGOs and metal thin films, as shown in Table 2. In addition, the composite foam heaters reached higher temperature than the typical commercial metal electrothermal heater $\mathrm{Fe}-\mathrm{Cr}-\mathrm{Al}$ and $\mathrm{Ni}-\mathrm{Cr}$ alloy plates at the same input power density (Fig. 4e, f). In other words, the input power required for the composite foams was less than that of the commercial alloy-based heaters in order to reach the same temperature with the same heater area. More intriguingly, arising from the uniform distribution of MXene on PI skeleton, the lightweight C-MXene@PI composite foam heaters had an even temperature distribution, which could not be achieved by the heavy commercial heaters composed of metal alloy strips which easily had local overheating (Fig. 4e, f). Based on the high electrothermal performance, a demonstration for a high-performance deicing or anti-icing application at low voltages is well shown (Fig. $3 \mathrm{~g}$, h). Combining the excellent flexibility, high heat performance, low working voltage and uniform temperature distribution under $12 \mathrm{~V}$ in the range of automobile power supply, and the robust behavior demonstrated by the life-time test, the C-MXene@ PI composite foam heaters show extended prospects in practical applications and can be produced on large scale for health-care-related products. The combination of the excellent MI shielding and electrothermal performance further efficiently demonstrates the great application potentials of the C-MXene@PI composites as multifunctional devices.

\subsection{Electromechanical Sensing Performance of the Composite Foam}

Since our C-MXene @PI composite foams are highly flexible and robust, as the second extension, they can be effortlessly attached to human body as wearable sensors for detecting the human motions [43-45]. As the three-dimensional (3D) composite samples were bent, stretching and compressing were caused in different sides (Fig. 5a). The stretched and compressed side of the 3D composite sensors corresponds to the formation of less and more conductive paths in the C-MXene@PI composites, respectively, which correspond to the increased and decreased electrical resistance, respectively (Fig. 5b-d). The gauge factor corresponding to the sensitivity of the foam sensors is also calculated based on the bending angle (Fig. S8), and we can conclude that the bending-induced stretching leads to a high sensitivity. When the composites bent to an angel and then kept steady, the resistance of the sensor initially changed due to the deformation and then kept stable. Moreover, a larger relative resistance change is observed for a larger bending angle,

Table 2 Comparison of $H_{\mathrm{p}}$ of typical heaters

\begin{tabular}{lllll}
\hline Heating material & Substrate & $H_{\mathrm{p}}\left[{ }^{\circ} \mathrm{C} \mathrm{cm}^{2} \mathrm{~W}^{-1}\right]$ & Refs. & Notes \\
\hline SWCNTs & Glass & 212,200 & {$[51,52]$} & Spray method \\
& $/$ & 137 & {$[67]$} & Dip-coating method \\
MWCNTs & PET & 94 & {$[68]$} & Yarn from vertical-aligned \\
rGO & Glass & 163 & {$[66]$} & Spin-coated GO/heat-treated \\
$\mathrm{Ag}$ & $/$ & 92 & & Silver paste \\
& & 54 & This work & Silver paste \\
$\mathrm{Pt}$ & none & 65 & & Sputtering \\
$\mathrm{Fe}-\mathrm{Cr}-\mathrm{Al}$ alloy & none & 85 & This study & Scalable dip-coating approach \\
Ni-Cr alloy & none & 62 & & \\
MXene & PI foam & 253 & &
\end{tabular}


(a)
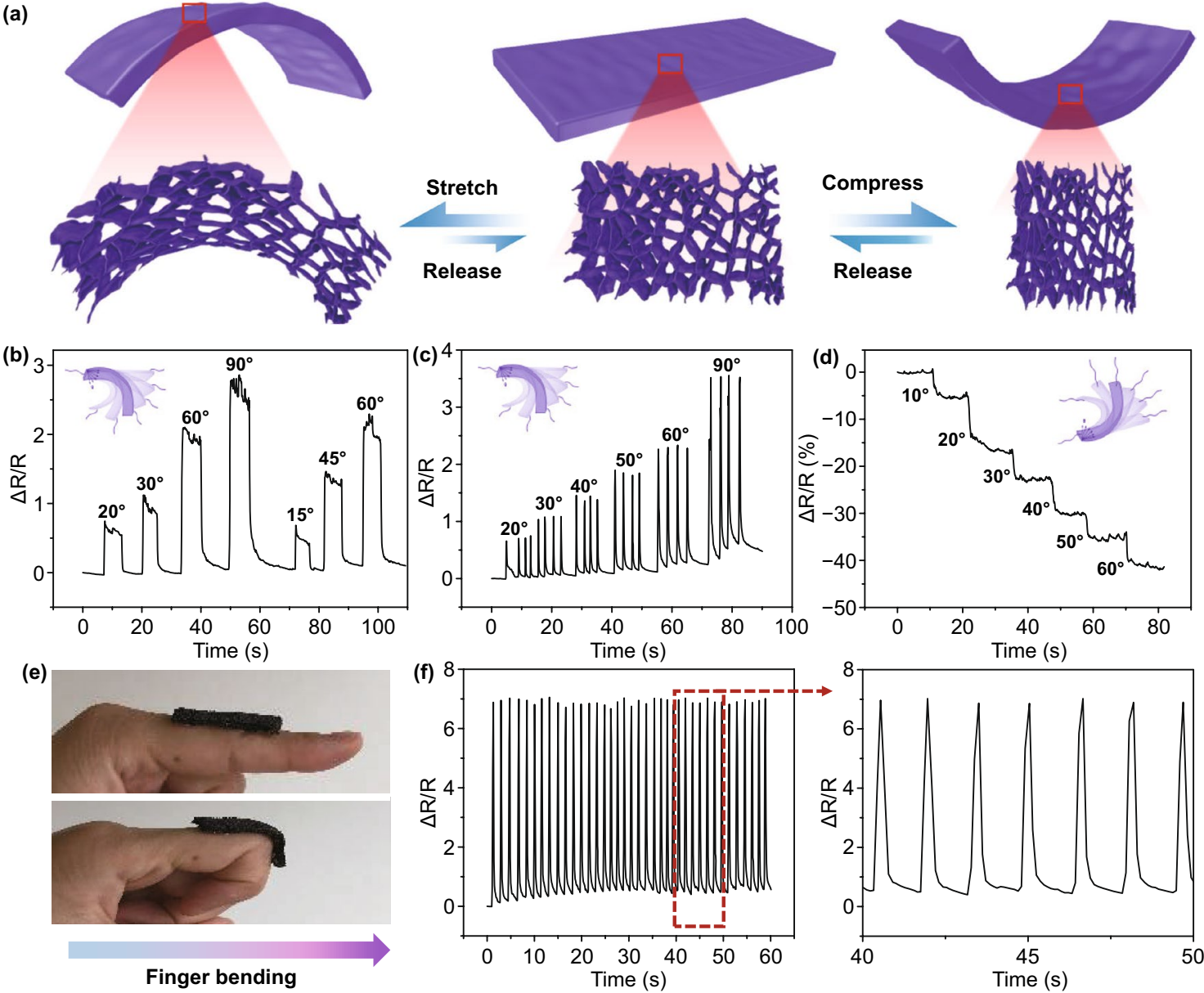

Compress
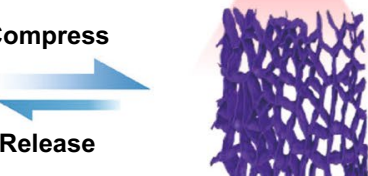

Release

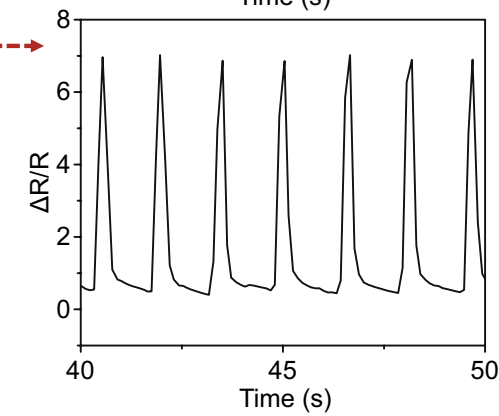

(g)

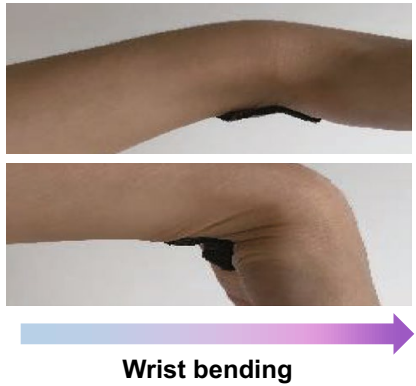

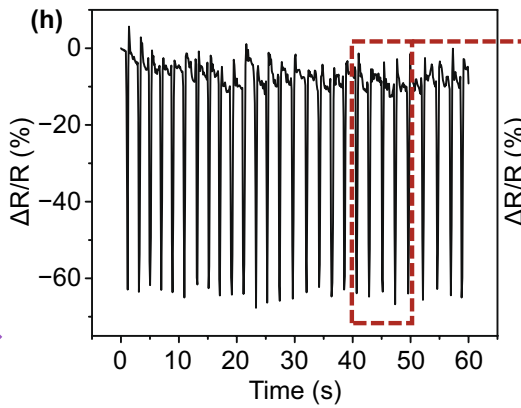

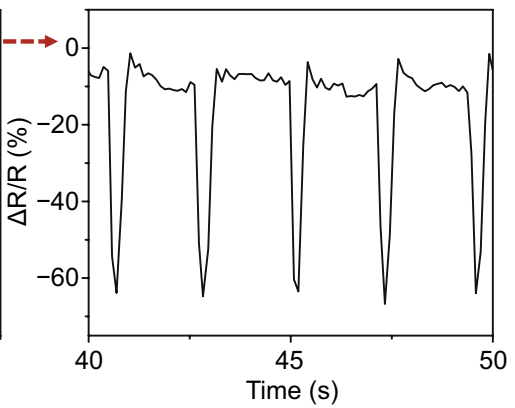

Fig. 5 Detections of human motions for the flexible C-MXene@PI composite foams as electromechanical strain sensors. a Bending-induced stretch and compression modes of the C-MXene@PI composite foams. b, $\mathbf{c}$ The resistance changes $(\Delta R / R=(R 1-R) / R$, where R1 and R are the resistance and initial resistance of the sample, resistance) of the C-MXene@PI composite foam upon various bending angle induced stretch. $\mathbf{d}$ The resistance changes of the C-MXene@PI composite foam upon various bending angle induced compress. e, $\mathbf{f}$ Detections of finger bending activities with cyclic bending which induces resistance change of the C-MXene@PI composite foam. $\mathbf{g}$, h Detections of wrist bending activities with cyclic bending which induces resistance change of the C-MXene@PI composite foam

showing the usefulness of our sensors in the detection of various human motions, from small strain to large strain activities. Additionally, even the bending speed of the sample was very fast, the change of the resistance could be well detected and distinguished. Therefore, the detected relatively resistance change's signal of the sensor attached on a finger bending circularly can be displayed. When the finger bent to a fixed angel, then returned periodically, the resistance of the C-MXene@PI composite foam sensor increased and recovered accordingly and periodically (Fig. 5e, f), ascribing to 
the stretching caused by the bend of the composite foams. In contrast, when the composite foam was attached on the wrist bending circularly, the resistance decreased and recovered periodically due to the compression caused by the bend of the composite foams (Fig. $5 \mathrm{~g}, \mathrm{~h}$ ). According to the measured electrical signals from the composite sensor, we can easily deduce that in the testing period, the finger or wrist repeatedly bends and moves back quickly with similar amplitude, and number and of bends in each interval.

\section{Conclusion}

Lightweight, ultra-flexible, and large-area C-MXene@PI composite foams were prepared in a facile and scalable dip-coating followed by a chemical crosslinking approach. The coactions of the highly porous yet robust PI scaffolds, coated MXene nanoflakes, and the effective chemical crosslink treatment rendered the C-MXene@PI foams with hydrophobicity, waterproof capability, anti-oxidation and extremetemperature stability. The combination also benefits high utilization of the MXene electrical conductivity, and an interfacial polarization between the MXene and PI. As-prepared C-MXene@PI foams show an ultrahigh EMI shielding performance accompanied by excellent durability and reliability. The 1.5-mm-thick C-MXene@PI composite foams exhibit a satisfactory X-band EMI SE of 22.5 to $62.5 \mathrm{~dB}$ at a density of 28.7 to $48.7 \mathrm{mg} \mathrm{cm}^{-3}$. Excellent SSE and $\mathrm{SSE} / d$ values of $1,971 \mathrm{~dB} \mathrm{~cm} \mathrm{~cm}^{-1}$ and $21,317 \mathrm{~dB} \mathrm{~cm}^{2} \mathrm{~g}^{-1}$ are achieved, respectively, for the C-MXene@PI foams, significantly surpassing other typical porous architectures. Furthermore, two possible extensions of the C-MXene@PI foams were exanimated. A rapid reproducible, and stable electrothermal effect of C-MXene@PI foams at low voltages is demonstrated, which shows ultrahigh heat performance and uniform heat distribution. Secondly, the excellent performance of the C-MXene@PI foams as flexible wearable sensors is well demonstrated with sensitive and reliable detection capability of human motions. The integration of ultrahigh EMI shielding, electrothermal, and electromechanical sensing performances of the C-MXene@PI foams as well as their facile and scalable production approach suggest the promising application potentials for next-generation flexible and multifunctional electronic devices and aerospace.
Acknowledgements Z.Z. appreciates the support of the Qilu Young Scholar Program of Shandong University (No. 31370082163127). Moreover, the authors acknowledge funding from the support from the Chinese Scholarship Council (to NW, project \# 201709370040).

Funding Open access funding provided by Shanghai Jiao Tong University.

Open Access This article is licensed under a Creative Commons Attribution 4.0 International License, which permits use, sharing, adaptation, distribution and reproduction in any medium or format, as long as you give appropriate credit to the original author(s) and the source, provide a link to the Creative Commons licence, and indicate if changes were made. The images or other third party material in this article are included in the article's Creative Commons licence, unless indicated otherwise in a credit line to the material. If material is not included in the article's Creative Commons licence and your intended use is not permitted by statutory regulation or exceeds the permitted use, you will need to obtain permission directly from the copyright holder. To view a copy of this licence, visit http://creativecommons.org/licenses/by/4.0/.

Supplementary Information The online version contains supplementary material available at https://doi.org/10.1007/ s40820-022-00800-0.

\section{References}

1. A. Iqbal, F. Shahzad, K. Hantanasirisakul, M.K. Kim, J. Kwon et al., Anomalous absorption of electromagnetic waves by $2 \mathrm{D}$ transition metal carbonitride $\mathrm{Ti}_{3} \mathrm{CNT}_{x}$ (MXene). Science 369(6502), 446-450 (2020). https://doi.org/10.1126/science. aba7977

2. Y. Zhang, Y. Huang, T. Zhang, H. Chang, P. Xiao et al., Broadband and tunable high-performance microwave absorption of an ultralight and highly compressible graphene foam. Adv. Mater. 27(12), 2049-2053 (2015). https://doi.org/10.1002/ adma.201405788

3. B. Wen, M. Cao, M. Lu, W. Cao, H. Shi et al., Reduced graphene oxides: light-weight and high-efficiency electromagnetic interference shielding at elevated temperatures. Adv. Mater. 26(21), 3484-3489 (2014). https://doi.org/10.1002/ adma. 201400108

4. Y. Yang, M.C. Gupta, K.L. Dudley, R.W. Lawrence, Conductive carbon nanofiber-polymer foam structures. Adv. Mater. 17(16), 1999-2003 (2005). https://doi.org/10.1002/adma. 200500615

5. Y. Yang, M.C. Gupta, K.L. Dudley, R.W. Lawrence, Novel carbon nanotube-polystyrene foam composites for electromagnetic interference shielding. Nano Lett. 5(11), 2131-2134 (2005). https://doi.org/10.1021/n1051375r

6. Z. Zeng, T. Wu, D. Han, Q. Ren, G. Siqueira et al., Ultralight, flexible, and biomimetic nanocellulose/silver nanowire 
aerogels for electromagnetic interference shielding. ACS Nano 14(3), 2927-2938 (2020). https://doi.org/10.1021/acsnano. $9 \mathrm{~b} 07452$

7. Z. Zeng, C. Wang, T. Wu, D. Han, M. Luković et al., Nanocellulose assisted preparation of ambient dried, large-scale and mechanically robust carbon nanotube foams for electromagnetic interference shielding. J. Mater. Chem. A 8(35), 1796917979 (2020). https://doi.org/10.1039/d0ta05961g

8. N. Yousefi, X. Sun, X. Lin, X. Shen, J. Jia et al., Highly aligned graphene/polymer nanocomposites with excellent dielectric properties for high-performance electromagnetic interference shielding. Adv. Mater. 26(31), 5480-5487 (2014). https://doi.org/10.1002/adma.201305293

9. N. Li, Y. Huang, F. Du, X. He, X. Lin et al., Electromagnetic interference (EMI) shielding of single-walled carbon nanotube epoxy composites. Nano Lett. 6(6), 1141-1145 (2006). https:// doi.org/10.1021/n10602589

10. Q. Wei, S. Pei, X. Qian, H. Liu, Z. Liu et al., Superhigh electromagnetic interference shielding of ultrathin aligned pristine graphene nanosheets film. Adv. Mater. 32(14), 1907411 (2020). https://doi.org/10.1002/adma.201907411

11. Q. Song, F. Ye, X. Yin, W. Li, H. Li et al., Carbon nanotubemultilayered graphene edge plane core-shell hybrid foams for ultrahigh-performance electromagnetic-interference shielding. Adv. Mater. 29(31), 1701583 (2017). https://doi.org/10.1002/ adma.201701583

12. P.C.P. Watts, W.K. Hsu, A. Barnes, B. Chambers, High permittivity from defective multiwalled carbon nanotubes in the X-Band. Adv. Mater. 15(7-8), 600-603 (2003). https://doi.org/ 10.1002/adma.200304485

13. Z. Zeng, F. Jiang, Y. Yue, D. Han, L. Lin et al., Flexible and ultrathin waterproof cellular membranes based on high-conjunction metal-wrapped polymer nanofibers for electromagnetic interference shielding. Adv. Mater. 32(19), 1908496 (2020). https://doi.org/10.1002/adma.201908496

14. B. Shen, W. Zhai, W. Zheng, Ultrathin flexible graphene film: an excellent thermal conducting material with efficient EMI shielding. Adv. Funct. Mater. 24(28), 4542-4548 (2014). https://doi.org/10.1002/adfm.201400079

15. D.X. Yan, H. Pang, B. Li, R. Vajtai, L. Xu et al., Structured reduced graphene oxide/polymer composites for ultra-efficient electromagnetic interference shielding. Adv. Funct. Mater. 25(4), 559-566 (2015). https://doi.org/10.1002/adfm.20140 3809

16. Z. Zeng, H. Jin, M. Chen, W. Li, L. Zhou et al., Lightweight and anisotropic porous MWCNT/WPU composites for ultrahigh performance electromagnetic interference shielding. Adv. Funct. Mater. 26(2), 303-310 (2016). https://doi.org/10.1002/ adfm.201503579

17. J. Liu, H.B. Zhang, R. Sun, Y. Liu, Z. Liu et al., Hydrophobic, flexible, and lightweight MXene foams for high-performance electromagnetic-interference shielding. Adv. Mater. 29(38), 1702367 (2017). https://doi.org/10.1002/adma.201702367

18. Z. Zeng, H. Jin, M. Chen, W. Li, L. Zhou et al., Microstructure design of lightweight, flexible, and high electromagnetic shielding porous multiwalled carbon nanotube/polymer composites. Small 13(34), 1701388 (2017). https://doi.org/10. 1002/smll.201701388

19. Z. Chen, C. Xu, C. Ma, W. Ren, H.M. Cheng, Lightweight and flexible graphene foam composites for high-performance electromagnetic interference shielding. Adv. Mater. 25(9), 1296-1300 (2013). https://doi.org/10.1002/adma.201204196

20. A. Iqbal, P. Sambyal, C.M. Koo, 2D MXenes for electromagnetic shielding: a review. Adv. Funct. Mater. 30(47), 2000883 (2020). https://doi.org/10.1002/adfm.202000883

21. Z. Fu, N. Wang, D. Legut, C. Si, Q. Zhang et al., Rational design of flexible two-dimensional MXenes with multiple functionalities. Chem. Rev. 119(23), 11980-12031 (2019). https://doi.org/10.1021/acs.chemrev.9b00348

22. F. Shahzad, M. Alhabeb, C.B. Hatter, B. Anasori, S.M. Hong et al., Electromagnetic interference shielding with 2D transition metal carbides (MXenes). Science 353(6304), 1137-1140 (2016). https://doi.org/10.1126/science.aag2421

23. M. Han, C.E. Shuck, R. Rakhmanov, D. Parchment, B. Anasori et al., Beyond $\mathrm{Ti}_{3} \mathrm{C}_{2} \mathrm{~T}_{\mathrm{x}}$ : MXenes for electromagnetic interference shielding. ACS Nano 14(4), 5008-5016 (2020). https:// doi.org/10.1021/acsnano.0c01312

24. J. Zhang, N. Kong, S. Uzun, A. Levitt, S. Seyedin et al., Scalable manufacturing of free-standing, strong $\mathrm{Ti}_{3} \mathrm{C}_{2} \mathrm{~T}_{\mathrm{x}}$ MXene films with outstanding conductivity. Adv. Mater. 32(23), 2001093 (2020). https://doi.org/10.1002/adma.202001093

25. D. Nepal, W.J. Kennedy, R. Pachter, R.A. Vaia, Toward architected nanocomposites: MXenes and beyond. ACS Nano 15(1), 21-28 (2021). https://doi.org/10.1021/acsnano.0c09834

26. Z. Zeng, C. Wang, G. Siqueira, D. Han, A. Huch et al., Nanocellulose-MXene biomimetic aerogels with orientationtunable electromagnetic interference shielding performance. Adv. Sci. 7(15), 2000979 (2020). https://doi.org/10.1002/advs. 202000979

27. Z. Zeng, E. Mavrona, D. Sacré, N. Kummer, J. Cao et al., Terahertz birefringent biomimetic aerogels based on cellulose nanofibers and conductive nanomaterials. ACS Nano 15(4), 7451-7462 (2021). https://doi.org/10.1021/acsnano.1c00856

28. H. Xu, X. Yin, X. Li, M. Li, S. Liang et al., Lightweight $\mathrm{Ti}_{2} \mathrm{CT}_{\mathrm{x}}$ MXene/Poly(vinyl alcohol) composite foams for electromagnetic wave shielding with absorption-dominated feature. ACS Appl. Mater. Interface 11(10), 10198-10207 (2019). https://doi.org/10.1021/acsami.8b21671

29. F. Xie, F. Jia, L. Zhuo, Z. Lu, L. Si et al., Ultrathin MXene/ aramid nanofiber composite paper with excellent mechanical properties for efficient electromagnetic interference shielding. Nanoscale 11(48), 23382-23391 (2019). https://doi.org/10. 1039/C9NR07331K

30. C. Weng, T. Xing, H. Jin, G. Wang, Z. Dai et al., Mechanically robust ANF/MXene composite films with tunable electromagnetic interference shielding performance. Comp. Part A Appl. Sci. Manuf. 135, 105927 (2020). https://doi.org/10. 1016/j.compositesa.2020.105927

31. W.T. Cao, F.F. Chen, Y.J. Zhu, Y.G. Zhang, Y.Y. Jiang et al., Binary strengthening and toughening of MXene/Cellulose nanofiber composite paper with nacre-inspired structure and superior electromagnetic interference shielding properties. 
ACS Nano 12(5), 4583-4593 (2018). https://doi.org/10.1021/ acsnano.8b00997

32. Q. Liu, Y. Zhang, Y. Liu, Z. Liu, B. Zhang et al., Ultrathin, biomimetic multifunctional leaf-like silver nanowires $/ \mathrm{Ti}_{3} \mathrm{C}_{2} \mathrm{~T}_{\mathrm{x}}$ MXene/cellulose nanofibrils nanocomposite film for high-performance electromagnetic interference shielding and thermal management. J. Alloys Compd. 860, 158151 (2021). https:// doi.org/10.1016/j.jallcom.2020.158151

33. Z. Zhan, Q. Song, Z. Zhou, C. Lu, Ultrastrong and conductive MXene/cellulose nanofiber films enhanced by hierarchical nano-architecture and interfacial interaction for flexible electromagnetic interference shielding. J. Mater. Chem. C 7(32), 9820-9829 (2019). https://doi.org/10.1039/c9tc03309b

34. W. Xin, G.Q. Xi, W.T. Cao, C. Ma, T. Liu et al., Lightweight and flexible MXene/CNF/silver composite membranes with a brick-like structure and high-performance electromagnetic-interference shielding. RSC Adv. 9(51), 29636-29644 (2019). https://doi.org/10.1039/c9ra06399d

35. M. Carey, M.W. Barsoum, MXene polymer nanocomposites: a review. Mater. Today Adv. 9, 100120 (2021). https://doi. org/10.1016/j.mtadv.2020.100120

36. X. Zhao, A. Vashisth, E. Prehn, W. Sun, S.A. Shah et al., Antioxidants unlock shelf-stable $\mathrm{Ti}_{3} \mathrm{C}_{2} \mathrm{~T}_{\mathrm{x}}$ (MXene) nanosheet dispersions. Matter 1(7), 513-526 (2019). https:// doi.org/10.1016/j.matt.2019.05.020

37. Y.J. Wan, K. Rajavel, X.M. Li, X.Y. Wang, S.Y. Liao et al., Electromagnetic interference shielding of $\mathrm{Ti}_{3} \mathrm{C}_{2} \mathrm{~T}_{\mathrm{x}}$ MXene modified by ionic liquid for high chemical stability and excellent mechanical strength. Chem. Eng. J. 408, 127303 (2021). https://doi.org/10.1016/j.cej.2020.127303

38. H. Chen, Y. Wen, Y. Qi, Q. Zhao, L. Qu et al., Pristine titanium carbide MXene films with environmentally stable conductivity and superior mechanical strength. Adv. Funct. Mater. 30(5), 1906996 (2019). https://doi.org/10.1002/adfm. 201906996

39. C. Tan, Z. Dong, Y. Li, H. Zhao, X. Huang et al., A high performance wearable strain sensor with advanced thermal management for motion monitoring. Nat. Commun. 11, 3530 (2020). https://doi.org/10.1038/s41467-020-17301-6

40. D. Kang, P.V. Pikhitsa, Y.W. Choi, C. Lee, S.S. Shin et al., Ultrasensitive mechanical crack-based sensor inspired by the spider sensory system. Nature 516, 222-226 (2014). https:// doi.org/10.1038/nature14002

41. X.J. Luo, L. Li, H.B. Zhang, S. Zhao, Y. Zhang et al., Multifunctional $\mathrm{Ti}_{3} \mathrm{C}_{2} \mathrm{~T}_{\mathrm{x}}$ MXene/low-density polyethylene soft robots with programmable configuration for amphibious motions. ACS Appl. Mater. Interfaces 13(38), 45833-45842 (2021). https://doi.org/10.1021/acsami.1c11056

42. Z. Zeng, S.I.S. Shahabadi, B. Che, Y. Zhang, C. Zhao et al., Highly stretchable, sensitive strain sensors with a wide linear sensing region based on compressed anisotropic graphene foam/polymer nanocomposites. Nanoscale 9(44), 17396 (2017). https://doi.org/10.1039/C7NR05106A

43. D. Song, X. Li, X. Li, X. Jia, P. Min et al., Hollow-structured MXene-PDMS composites as flexible, wearable and highly bendable sensors with wide working range. J. Colloid Interface
Sci. 555, 751-758 (2019). https://doi.org/10.1016/j.jcis.2019. 08.020

44. C.S. Boland, U. Khan, G. Ryan, S. Barwich, R. Charifou et al., Sensitive electromechanical sensors using viscoelastic graphene-polymer nanocomposites. Science 354(6317), 1257-1260 (2016). https://doi.org/10.1126/science.aag2879

45. Y. Wang, L. Wang, T. Yang, X. Li, X. Zang et al., Wearable and highly sensitive graphene strain sensors for human motion monitoring. Adv. Funct. Mater. 24(29), 4666-4670 (2014). https://doi.org/10.1002/adfm.201400379

46. Z. Xiang, Y. Shi, X. Zhu, L. Cai, W. Lu, Flexible and waterproof 2D/1D/0D construction of MXene-based nanocomposites for electromagnetic wave absorption, EMI shielding, and photothermal conversion. Nano-Micro Lett. 13, 150 (2021). https://doi.org/10.1007/s40820-021-00673-9

47. D. Jiao, F. Lossada, J. Guo, O. Skarsetz, D. Hoenders et al., Electrical switching of high-performance bioinspired nanocellulose nanocomposites. Nat. Commun. 12, 1312 (2021). https://doi.org/10.1038/s41467-021-21599-1

48. L. Zhang, M. Baima, T.L. Andrew, Transforming commercial textiles and threads into sewable and weavable electric heaters. ACS Appl. Mater. Interface 9(37), 32299-32307 (2017). https://doi.org/10.1021/acsami.7b10514

49. Y. Guo, C. Dun, J. Xu, J. Mu, P. Li et al., Ultrathin, washable, and large-area graphene papers for personal thermal management. Small 13(44), 1702645 (2017). https://doi.org/10.1002/ smll.201702645

50. Y. Yao, K.K. Fu, C. Yan, J. Dai, Y. Chen et al., Three-dimensional printable high-temperature and high-rate heaters. ACS Nano 10(5), 5272-5279 (2016). https://doi.org/10.1021/acsna no.6b01059

51. Y.H. Yoon, J.W. Song, D. Kim, J. Kim, J.K. Park et al., Transparent film heater using single-walled carbon nanotubes. Adv. Mater. 19(23), 4284-4287 (2007). https://doi.org/10.1002/ adma. 200701173

52. J.J. Bae, S.C. Lim, G.H. Han, Y.W. Jo, D.L. Doung et al., Heat dissipation of transparent graphene defoggers. Adv. Funct. Mater. 22(22), 4819-4826 (2012). https://doi.org/10.1002/ adfm.201201155

53. Z. Zeng, M. Chen, H. Jin, W. Li, X. Xue et al., m Thin and flexible multi-walled carbon nanotube/waterborne polyurethane composites with high-performance electromagnetic interference shielding. Carbon 96, 768-777 (2016). https:// doi.org/10.1016/j.carbon.2015.10.004

54. A. Zhou, Y. Liu, S. Li, X. Wang, G. Ying et al., From structural ceramics to $2 \mathrm{D}$ materials with multi-applications: a review on the development from max phases to MXenes. J. Adv. Ceram. 10(6), 1194-1242 (2021). https://doi.org/10. 1007/s40145-021-0535-5

55. M. Wu, M. He, Q. Hu, Q. Wu, G. Sun et al., $\mathrm{Ti}_{3} \mathrm{C}_{2}$ MXenebased sensors with high selectivity for $\mathrm{NH}_{3}$ detection at room temperature. ACS Sens. 4(10), 2763-2770 (2019). https://doi. org/10.1021/acssensors.9b01308

56. J. Liu, H.B. Zhang, X. Xie, R. Yang, Z. Liu et al., Multifunctional, superelastic, and lightweight MXene/polyimide 
aerogels. Small 14(45), 1802479 (2018). https://doi.org/10. 1002/smll.201802479

57. N. Wu, Z. Zeng, N. Kummer, D. Han, R. Zenobi et al., Ultrafine cellulose nanofiber-assisted physical and chemical cross-linking of mxene sheets for electromagnetic interference shielding. Small Methods 5(12), 2100889 (2021). https://doi. org/10.1002/smtd.202100889

58. J.M. Thomassin, C. Jérôme, T. Pardoen, C. Bailly, I. Huynen et al., Polymer/carbon based composites as electromagnetic interference (EMI) shielding materials. Mater. Sci. Eng. R Rep. 74(7), 211-232 (2013). https://doi.org/10.1016/j.mser. 2013.06.001

59. J. Ma, M. Zhan, K. Wang, Ultralightweight silver nanowires hybrid polyimide composite foams for high-performance electromagnetic interference shielding. ACS Appl. Mater. Interface 7(1), 563-576 (2015). https://doi.org/10.1021/am506 7095

60. Y.Y. Wang, Z.H. Zhou, C.G. Zhou, W.J. Sun, J.F. Gao et al., Lightweight and robust carbon nanotube/polyimide foam for efficient and heat-resistant electromagnetic interference shielding and microwave absorption. ACS Appl. Mater. Interface 12(7), 8704-8712 (2020). https://doi.org/10.1021/acsami. $9 \mathrm{~b} 21048$

61. J. Ling, W. Zhai, W. Feng, B. Shen, J. Zhang et al., Facile preparation of lightweight microcellular polyetherimide/graphene composite foams for electromagnetic interference shielding. ACS Appl. Mater. Interface 5(7), 2677-2684 (2013). https:// doi.org/10.1021/am303289m

62. G. Sang, P. Xu, T. Yan, V. Murugadoss, N. Naik et al., Interface engineered microcellular magnetic conductive polyurethane nanocomposite foams for electromagnetic interference shielding. Nano-Micro Lett. 13, 153 (2021). https://doi.org/ 10.1007/s40820-021-00677-5

63. X. Wu, T. Tu, Y. Dai, P. Tang, Y. Zhang et al., Direct ink writing of highly conductive MXene frames for tunable electromagnetic interference shielding and electromagnetic waveinduced thermochromism. Nano-Micro Lett. 13, 148 (2021). https://doi.org/10.1007/s40820-021-00665-9

64. J.Q. Luo, S. Zhao, H.B. Zhang, Z. Deng, L. Li et al., Flexible, stretchable and electrically conductive MXene/natural rubber nanocomposite films for efficient electromagnetic interference shielding. Compos. Sci. Technol. 182, 107754 (2019). https:// doi.org/10.1016/j.compscitech.2019.107754

65. Y. Li, X. Pei, B. Shen, W. Zhai, L. Zhang et al., Polyimide/ graphene composite foam sheets with ultrahigh thermostability for electromagnetic interference shielding. RSC Adv. 5(31), 24342-24351 (2015). https://doi.org/10.1039/C4RA16421K

66. D. Sui, Y. Huang, L. Huang, J. Liang, Y. Ma et al., Flexible and transparent electrothermal film heaters based on graphene materials. Small 7(22), 3186-3192 (2011). https://doi.org/10. 1002/smll.201101305

67. T.J. Kang, T. Kim, S.M. Seo, Y.J. Park, Y.H. Kim, Thicknessdependent thermal resistance of a transparent glass heater with a single-walled carbon nanotube coating. Carbon 49(4), 1087-1093 (2011). https://doi.org/10.1016/j.carbon.2010.11. 012

68. H.S. Jang, S.K. Jeon, S.H. Nahm, The manufacture of a transparent film heater by spinning multi-walled carbon nanotubes. Carbon 49(1), 111-116 (2011). https://doi.org/10.1016/j.carbon.2010.08.049

69. H. Yang, Z. Yu, P. Wu, H. Zou, P. Liu, Electromagnetic interference shielding effectiveness of microcellular polyimide/ in situ thermally reduced graphene oxide/carbon nanotubes nanocomposites. Appl. Surf. Sci. 434, 318-325 (2018). https:// doi.org/10.1016/j.apsusc.2017.10.191

70. Y.Y. Wang, W.J. Sun, D.X. Yan, K. Dai, Z.M. Li, Ultralight carbon nanotube/graphene/polyimide foam with heterogeneous interfaces for efficient electromagnetic interference shielding and electromagnetic wave absorption. Carbon 176, 118-125 (2021). https://doi.org/10.1016/j.carbon.2020.12.028

71. Z. Yu, T. Dai, S. Yuan, H. Zou, P. Liu, Electromagnetic interference shielding performance of anisotropic polyimide/graphene composite aerogels. ACS Appl. Mater. Interface 12(27), 30990-31001 (2020). https://doi.org/10.1021/acsami.0c07122

72. H. Yang, Z. Li, H. Zou, P. Liu, Preparation of porous polyimide/in-situ reduced graphene oxide composite films for electromagnetic interference shielding. Poly. Adv. Technol. 28(2), 233-242 (2017). https://doi.org/10.1002/pat.3879

73. Z. Li, Z. Lin, M. Han, Y. Zhang, J. Yu, Vertical graphene nanosheet/polyimide composite films for electromagnetic interference shielding. ACS Appl. Nano Mater. 4(7), 74617470 (2021). https://doi.org/10.1021/acsanm.1c01471

74. L. Nayak, T.K. Chaki, D. Khastgir, Electrical percolation behavior and electromagnetic shielding effectiveness of polyimide nanocomposites filled with carbon nanofibers. J. Appl. Polym. Sci. 131(24), 40914 (2014). https://doi.org/10.1002/ app.40914

75. D.Y. Kong, J. Li, A.R. Guo, X.L. Xiao, High temperature electromagnetic shielding shape memory polymer composite. Chem. Eng. J. 408, 127365 (2021). https://doi.org/10.1016/j. cej.2020.127365

76. J. Li, Y. Ding, N. Yu, Q. Gao, X. Fan et al., Lightweight and stiff carbon foams derived from rigid thermosetting polyimide foam with superior electromagnetic interference shielding performance. Carbon 158, 45-54 (2020). https://doi.org/10. 1016/j.carbon.2019.11.075

77. Y. Li, B. Shen, X. Pei, Y. Zhang, D. Yi et al., Ultrathin carbon foams for effective electromagnetic interference shielding. Carbon 100, 375-385 (2016). https://doi.org/10.1016/j.carbon. 2016.01.030

78. Y. Cheng, X. Li, Y. Qin, Y. Fang, G. Liu et al., Hierarchically porous polyimide/ $\mathrm{Ti}_{3} \mathrm{C}_{2} \mathrm{~T}_{\mathrm{X}}$ film with stable electromagnetic interference shielding after resisting harsh conditions. Sci. Adv. (2021). https://doi.org/10.1126/sciadv.abj1663 